\title{
ASYMPTOTIC BEHAVIOR OF SOLUTIONS TO A CLASS OF SEMILINEAR PARABOLIC EQUATIONS WITH BOUNDARY DEGENERACY
}

\author{
CHUNPENG WANG
}

(Communicated by James E. Colliander)

\begin{abstract}
This paper concerns the asymptotic behavior of solutions to a semilinear parabolic equation with boundary degeneracy. It is proved that for the problem in a bounded domain with a homogeneous boundary condition, there exist both nontrivial global and blowing-up solutions if the degeneracy is not strong, while the nontrivial solution must blow up in a finite time if the degeneracy is strong enough. For the problem in an unbounded domain, blowing-up theorems of Fujita type are established and the critical Fujita exponent is finite in the not strong degeneracy case, while infinite in the other case. Furthermore, the behavior of solutions at the degenerate point is studied, and it is shown that for the nontrivial initial datum vanishing at the degenerate point, the solution always vanishes at the degenerate point if the degeneracy is strong enough, while never if it is not.
\end{abstract}

\section{INTRODUCTION}

This paper concerns the equation of the form

$$
\frac{\partial u}{\partial t}=\frac{\partial}{\partial x}\left(x^{\lambda} \frac{\partial u}{\partial x}\right)+f(x, t, u), \quad 0<x<1, t>0,
$$

where $\lambda>0$. As a typical equation with boundary degeneracy, (1.1) is degenerate at $x=0$, a portion of the lateral boundary. Equations with degeneracy similar to the one in (1.1) are used to describe some models, such as the Black-Scholes model coming from the option pricing problem ([2] and hundreds of related papers), the Budyko-Sellers climate model ([12]) and a simplified Crocco-type equation coming from the study on the velocity field of a laminar flow on a flat plate ([6]). In recent years, the null controllability of the control system governed by (1.1) was studied. Particularly, for the control system governed by

$$
\frac{\partial u}{\partial t}=\frac{\partial}{\partial x}\left(x^{\lambda} \frac{\partial u}{\partial x}\right)+h(x, t) \chi_{\left(\omega_{1}, \omega_{2}\right)}, \quad(x, t) \in(0,1) \times(0, T),
$$

it is proved that the control system is null controllable if $0<\lambda<2$ ([1, 6, 7, 11, 14]), while not if $\lambda \geq 2([5])$, where $h$ is the control function and $\chi_{\left(\omega_{1}, \omega_{2}\right)}$ is the characteristic function of $\left(\omega_{1}, \omega_{2}\right)$ with $0<\omega_{1}<\omega_{2}<1$. Although the system can be not null controllable, [3, 4, 5] and [13] proved that the system is regional

Received by the editors May 21, 2011 and, in revised form, November 14, 2011.

2010 Mathematics Subject Classification. Primary 35K65, 35D30, 35B33.

Key words and phrases. Boundary degeneracy, asymptotic behavior.

The author was supported by the NNSF (grants No. 11071100 and 11222106), FANEDD of China and 985 Program of Jilin University. 
null controllable (and also persistent regional null controllable) and approximately controllable in $L^{2}((0,1))$, respectively, for each $\lambda>0$.

In this paper, we investigate the asymptotic behavior of solutions to (1.1) with the power-like source. Precisely, we consider the problem as follows:

$$
\begin{array}{ll}
\frac{\partial u}{\partial t}=\frac{\partial}{\partial x}\left(x^{\lambda} \frac{\partial u}{\partial x}\right)+u^{p}, & 0<x<1, t>0, \\
x^{\lambda} \frac{\partial u}{\partial x}(0, t)=0, \quad u(1, t)=0, & t>0, \\
u(x, 0)=u_{0}(x), & 0<x<1,
\end{array}
$$

where $\lambda>0, p>1$ and $u_{0}$ is nonnegative. In the nondegenerate case $\lambda=0$, it is well known that there exist both nontrivial global and blowing-up solutions to problem (1.2) -(1.4). However, it is shown in this paper that the conclusion is valid just for the case $0 \leq \lambda<2$. If the degeneracy is so strong that $\lambda \geq 2$, then the solution to problem (1.2) - (1.4) must blow up in a finite time for any nontrivial $u_{0}$. This is a new phenomenon of nonexistence of nontrivial global solutions to the parabolic problem in a bounded domain where there is a power-like source and a homogeneous boundary condition $([8,10])$. Since there are a homogeneous Neumann boundary condition at the boundary $x=0$ and a homogeneous Dirichlet boundary condition at the boundary $x=1$, it seems that $x=0$ should be a blowing-up point. To our surprise, it is shown that in the case $\lambda \geq 2$, the solution always vanishes at $x=0$ if $u_{0}(0)=0$. Contrarily, this phenomenon cannot happen in the case $0<\lambda<2$ unless $u$ is trivial. So, the degeneracy of the equation essentially affects the asymptotic behavior of solutions; further, there is a mutation for the asymptotic behavior of solutions at $\lambda=2$.

After the problem in a bounded interval, we are also interesting in the problem in an unbounded interval:

$$
\begin{array}{ll}
\frac{\partial u}{\partial t}=\frac{\partial}{\partial x}\left(x^{\lambda} \frac{\partial u}{\partial x}\right)+u^{p}, & x>0, t>0, \\
x^{\lambda} \frac{\partial u}{\partial x}(0, t)=0, & t>0, \\
u(x, 0)=u_{0}(x), & x>0,
\end{array}
$$

where $\lambda>0, p>1$ and $u_{0}$ is nonnegative. For problem (1.5)-(1.7), it is shown that the critical Fujita exponent is

$$
p_{c}=\left\{\begin{array}{cl}
3-\lambda, & \text { if } 0<\lambda<2, \\
+\infty, & \text { if } \lambda \geq 2
\end{array}\right.
$$

As an important topic for nonlinear partial differential equations, a critical Fujita exponent was begun in 1966 by Fujita [9] and there have been a number of extensions of Fujita's results in several directions since then, including similar results for numerous quasilinear parabolic equations and systems in various geometries with nonlinear sources or nonhomogeneous boundary conditions (see the survey papers [8, 10]). The results in this paper show that the critical Fujita exponent to problem (1.5) (1.7) is discontinuous at $\lambda=2$; further, the critical Fujita exponent is finite if $0<\lambda<2$, while infinite if $\lambda \geq 2$. Like the solution to problem (1.2)-(1.4), the solution to problem (1.5) (1.7) also possesses the following property if $u_{0}(0)=0$ : the solution always vanishes at $x=0$ in the case $\lambda \geq 2$, while the solution never vanishes at $(0, t)$ for any $t>0$ unless $u$ is trivial in the case $0<\lambda<2$. 
The paper is organized as follows. In $\S 2$, we introduce the main results of the paper. Problem (1.2)-(1.4) and problem (1.5)-(1.7) are studied in $\S 3$ and $\S 4$, respectively. Finally, in $\S 5$ we state similar results for the problems with inner degeneracy.

\section{MAin Results}

As in [17, solutions to problem (1.2)-(1.4) and problem (1.5)-(1.7) can be defined as follows.

Definition 2.1. Assume that $0<T \leq+\infty$. A nonnegative function $u$ is called a subsolution to problem (1.2)-(1.4) in $(0, T)$ if

(i) For any $0<\tilde{T}<T, u \in L^{\infty}((0,1) \times(0, \tilde{T}))$ with $\frac{\partial u}{\partial t} \in L^{2}((0,1) \times(0, \tilde{T}))$ and $x^{\lambda / 2} \frac{\partial u}{\partial x} \in L^{2}((0,1) \times(0, \tilde{T}))$.

(ii) For any $0<\tilde{T}<T$ and any nonnegative $\varphi \in C^{1}([0,1] \times[0, \tilde{T}])$ vanishing at $x=1$,

$$
\int_{0}^{\tilde{T}} \int_{0}^{1}\left(\frac{\partial u}{\partial t}(x, t) \varphi(x, t)+x^{\lambda} \frac{\partial u}{\partial x}(x, t) \frac{\partial \varphi}{\partial x}(x, t)\right) d x d t \leq \int_{0}^{\tilde{T}} \int_{0}^{1} u^{p}(x, t) \varphi(x, t) d x d t .
$$

(iii) $u(1, \cdot) \leq 0$ in $(0, T)$ and $u(\cdot, 0) \leq u_{0}(\cdot)$ in $(0,1)$ in the sense of trace.

Definition 2.2. Assume that $0<T \leq+\infty$. A nonnegative function $u$ is called a subsolution to problem (1.5) $-(1.7)$ in $(0, T)$ if

(i) For any $0<\tilde{T}<T, u \in L^{\infty}((0,+\infty) \times(0, \tilde{T}))$ with $\frac{\partial u}{\partial t} \in L^{2}((0, R) \times(0, \tilde{T}))$ and $x^{\lambda / 2} \frac{\partial u}{\partial x} \in L^{2}((0, R) \times(0, \tilde{T}))$ for each $R>0$.

(ii) For any $0<\tilde{T}<T$ and any nonnegative $\varphi \in C^{1}([0,+\infty) \times[0, \tilde{T}])$ vanishing when $x$ is large,

$$
\int_{0}^{\tilde{T}} \int_{0}^{+\infty}\left(\frac{\partial u}{\partial t}(x, t) \varphi(x, t)+x^{\lambda} \frac{\partial u}{\partial x}(x, t) \frac{\partial \varphi}{\partial x}(x, t)\right) d x d t \leq \int_{0}^{\tilde{T}} \int_{0}^{+\infty} u^{p}(x, t) \varphi(x, t) d x d t .
$$

(iii) $u(\cdot, 0) \leq u_{0}(\cdot)$ in $(0,+\infty)$ in the sense of trace.

Supersolutions can be defined similarly. A solution means that it is both a subsolution and a supersolution.

Proposition 2.1. (i) For any $0 \leq u_{0} \in L^{\infty}((0,1))$ with $x^{\lambda / 2} u_{0}^{\prime} \in L^{2}((0,1))$, there is a solution to problem (1.2)-(1.4) locally in time.

(ii) Assume that $\underline{u}$ and $\bar{u}$ are a subsolution and a supersolution to problem (1.2) (1.4) in $(0, T)$, respectively. Then $\underline{u} \leq \bar{u}$ in $(0,1) \times(0, T)$.

Proposition 2.2. (i) For any $0 \leq u_{0} \in L^{\infty}((0,+\infty))$ with $x^{\lambda / 2} u_{0}^{\prime} \in L^{2}((0, R))$ for each $R>0$, there is a solution to problem (1.5)-(1.7) locally in time.

(ii) Assume that $\underline{u}$ and $\bar{u}$ are a subsolution and a supersolution to problem (1.5)(1.7) in $(0, T)$, respectively. Then $\underline{u} \leq \bar{u}$ in $(0,+\infty) \times(0, T)$.

Proposition 2.1 can be proved similar to the nondegenerate case $\lambda=0$. The local existence is based on the parabolic regularization method and the uniform supersolutions and energy estimates for the regularized problems. The comparison principle follows by choosing $\varphi=(\underline{u}-\bar{u})_{+}$in the definition, where $s_{+}=\max \{s, 0\}$. For Proposition 2.2, the local existence is obtained by approximating the problem in an unbounded domain from the problems in bounded domains (see [15, Theorem 1.3.4), while the comparison principle is proved by a duality argument (see [15], Theorem 1.3.1). 
If $u$ is a solution to problem (1.2)-(1.4) (or problem (1.5)-(1.7)) in $(0,+\infty)$, we say that $u$ is a global solution in time. Otherwise, there exists $T>0$ such that $u$ is a solution in $(0, T)$ and satisfies

$$
\lim _{t \rightarrow T^{-}} \sup _{(0,1)} u(\cdot, t)=+\infty, \quad\left(\text { or } \lim _{t \rightarrow T^{-}} \sup _{(0,+\infty)} u(\cdot, t)=+\infty\right),
$$

and we say that $u$ blows up in a finite time.

The main results of problem (1.2)-(1.4) and problem (1.5)-(1.7) are as follows.

Theorem 2.1. Assume that $0<\lambda<2$. Then there exist both nontrivial global and blowing-up solutions to problem (1.2)-(1.4).

Theorem 2.2. Assume that $\lambda \geq 2$. Then the solution to problem (1.2)-(1.4) must blow up in a finite time for any nontrivial $0 \leq u_{0} \in L^{\infty}((0,1))$ with $x^{\lambda / 2} u_{0}^{\prime} \in$ $L^{2}((0,1))$.

Theorem 2.3. Assume that $u$ is the solution to problem (1.2)-(1.4) in $(0, T)$ $(0<T \leq+\infty)$ for nontrivial $0 \leq u_{0} \in C^{2}([0,1])$ with $u_{0}(0)=0$.

(i) If $0<\lambda<2$, then

$$
\lim _{x \rightarrow 0^{+}} u(x, t)>0, \quad 0<t<T .
$$

(ii) If $\lambda \geq 2$, then for any $0<t<T$, there exists $M(t)>0$ such that

$$
0 \leq u(x, t) \leq M(t) x, \quad 0<x<1 .
$$

Theorem 2.4. Assume that $0<\lambda<2$.

(i) If $1<p<3-\lambda$, then the solution to problem (1.5) (1.7) must blow up in a finite time for any nontrivial $0 \leq u_{0} \in L^{\infty}((0,+\infty))$ with $x^{\lambda / 2} u_{0}^{\prime} \in L^{2}((0, R))$ for each $R>0$.

(ii) If $p=3-\lambda$, then the solution to problem (1.5) -(1.7) must blow up in a finite time for any nontrivial $0 \leq u_{0} \in L^{\infty}((0,+\infty))$ with $x^{\lambda / 2} u_{0}^{\prime} \in L^{2}((0, R))$ for each $R>0$.

(iii) If $p>3-\lambda$, then there exist both nontrivial global and blowing-up solutions to problem (1.5) (1.7).

Theorem 2.5. Assume that $\lambda \geq 2$. Then the solution to problem (1.5) (1.7) must blow up in a finite time for any nontrivial $0 \leq u_{0} \in L^{\infty}((0,+\infty))$ with $x^{\lambda / 2} u_{0}^{\prime} \in L^{2}((0, R))$ for each $R>0$.

Theorem 2.6. Assume that $u$ is the solution to problem (1.5)-(1.7) in $(0, T)$ $(0<T \leq+\infty)$ for nontrivial $0 \leq u_{0} \in C^{2}([0,+\infty)) \cap L^{\infty}((0,+\infty))$ with $u_{0}(0)=0$.

(i) If $0<\lambda<2$, then

$$
\lim _{x \rightarrow 0^{+}} u(x, t)>0, \quad 0<t<T .
$$

(ii) If $\lambda \geq 2$, then for any $0<t<T$, there exists $M(t)>0$ such that

$$
0 \leq u(x, t) \leq M(t) x, \quad x>0 .
$$




\section{Problems in a bounded interval}

In this section, we study problem (1.2)-(1.4) and prove Theorems 2.12 .3

Proof of Theorem 2.1. First consider the global case. For $M>0$, set

$$
\bar{u}(x, t)=(t+M)^{-1 /(p-1)} w\left((t+M)^{-1 /(2-\lambda)} x\right), \quad 0<x<1, t>0 .
$$

A direct calculation shows that $\bar{u}$ is a supersolution to (1.2) if

$$
w \in C^{2}\left(\left(0, M^{-1 /(2-\lambda)}\right)\right)
$$

satisfies

$$
\left(r^{\lambda} w^{\prime}(r)\right)^{\prime}+\frac{1}{2-\lambda} r w^{\prime}(r)+\frac{1}{p-1} w(r)+w^{p}(r) \leq 0, \quad 0<r<M^{-1 /(2-\lambda)} .
$$

Take

$$
w(r)=\frac{1}{2-\lambda}\left(\frac{1}{M}-r^{2-\lambda}\right), \quad 0<r<M^{-1 /(2-\lambda)} .
$$

Then,

$$
\begin{aligned}
& \left(r^{\lambda} w^{\prime}(r)\right)^{\prime}+\frac{1}{2-\lambda} r w^{\prime}(r)+\frac{1}{p-1} w(r)+w^{p}(r) \\
& =-1-\frac{1}{2-\lambda} r^{2-\lambda}+\frac{1}{(p-1)(2-\lambda)}\left(\frac{1}{M}-r^{2-\lambda}\right)+\frac{1}{(2-\lambda)^{p}}\left(\frac{1}{M}-r^{2-\lambda}\right)^{p} \\
& 0<r<M^{-1 /(2-\lambda)}
\end{aligned}
$$

Therefore, $\bar{u}$ is a supersolution to (1.2) for each $M \geq M_{0}$, where

$$
M_{0}=\frac{1}{(p-1)(2-\lambda)}+\frac{1}{(2-\lambda)^{p}}+1
$$

Noting that $\lim _{r \rightarrow 0^{+}} r^{\lambda} w^{\prime}(r)=0$, one gets that $\bar{u}$ is a supersolution to problem (1.2) -(1.4) if

$$
u_{0}(x) \leq \bar{u}(x, 0), \quad 0<x<1 \quad\left(M \geq M_{0}\right) .
$$

The comparison principle (Proposition 2.1 (ii)) yields that the solution to problem (1.2) - (1.4) exists globally in time if $u_{0}$ satisfies (3.1).

Now let us turn to the blowing-up case. Set

$$
\psi(x)= \begin{cases}2, & \text { if } 0<x \leq \frac{1}{2} \\ 1+\cos (2 x-1) \pi, & \text { if } \frac{1}{2}<x \leq 1 .\end{cases}
$$

It is clear that $\psi \in C^{1}([0,1])$ is piecewise smooth and satisfies $\psi^{\prime}(0)=0, \psi(1)=0$ and

$$
\left(x^{\lambda} \psi^{\prime}(x)\right)^{\prime}=-2 \lambda \pi x^{\lambda-1} \sin (2 x-1) \pi-4 \pi^{2} x^{\lambda} \cos (2 x-1) \pi \geq-4 \pi^{2} \psi(x), \quad \frac{1}{2}<x \leq 1 .
$$


Assume that $u$ is a solution to problem (1.2)-(1.4) in $(0,+\infty)$. Then, it follows from Definition 2.1 that $u$ satisfies

$$
\begin{aligned}
& \frac{d}{d t} \int_{0}^{1} u(x, t) \psi(x) d x=\int_{0}^{1} u(x, t)\left(x^{\lambda} \psi^{\prime}(x)\right)^{\prime} d x+\int_{0}^{1} u^{p}(x, t) \psi(x) d x \\
& \quad \geq-4 \pi^{2} \int_{0}^{1} u(x, t) \psi(x) d x+\left(\int_{0}^{1} \psi(x) d x\right)^{1-p}\left(\int_{0}^{1} u(x, t) \psi(x) d x\right)^{p} \\
& \quad \geq-4 \pi^{2} \int_{0}^{1} u(x, t) \psi(x) d x+2^{1-p}\left(\int_{0}^{1} u(x, t) \psi(x) d x\right)^{p}, \quad t>0
\end{aligned}
$$

Therefore, if $u_{0}$ is sufficiently large such that

$$
\left(\int_{0}^{1} u_{0}(x) \psi(x) d x\right)^{p-1} \geq 2^{p+2} \pi^{2}
$$

then

$$
\frac{d}{d t} \int_{0}^{1} u(x, t) \psi(x) d x \geq 2^{-p}\left(\int_{0}^{1} u(x, t) \psi(x) d x\right)^{p}, \quad t>0 .
$$

Hence, there exists $T>0$ such that

$$
\lim _{t \rightarrow T^{-}} \int_{0}^{1} u(x, t) \psi(x) d x=+\infty
$$

which leads to

$$
\lim _{t \rightarrow T^{-}} \sup _{(0,1)} u(\cdot, t)=+\infty .
$$

Proof of Theorem 2.2. For $0<\delta<1$, set

$$
\psi_{\delta}(x)= \begin{cases}\frac{\lambda-1}{\delta} 2^{\lambda-1-\delta} x^{-\delta}-\frac{\lambda-1-\delta}{\delta} 2^{\lambda-1}-1, & \text { if } 0<x<\frac{1}{2}, \\ x^{1-\lambda}-1, & \text { if } \frac{1}{2} \leq x \leq 1 .\end{cases}
$$

It is clear that $\psi_{\delta} \in C^{1}((0,1])$ and $\psi_{\delta}$ is piecewise smooth with

$$
\left(x^{\lambda} \psi_{\delta}^{\prime}(x)\right)^{\prime}= \begin{cases}-(\lambda-1)(\lambda-1-\delta) 2^{\lambda-1-\delta} x^{\lambda-2-\delta}, & \text { if } 0<x<\frac{1}{2} \\ 0, & \text { if } \frac{1}{2} \leq x<1 .\end{cases}
$$

Therefore, there exists $C_{1}>0$ depending only on $\lambda$ but independent of $\delta$ such that

$$
\left(x^{\lambda} \psi_{\delta}^{\prime}(x)\right)^{\prime} \geq-C_{1} \delta \psi_{\delta}(x), \quad 0<x<1 .
$$

Additionally, a simple calculation shows that $\psi_{\delta} \in L^{1}((0,1))$ satisfies

$$
\int_{0}^{1} \psi_{\delta}(x) d x \leq C_{2}
$$

with some $C_{2}>0$ depending only on $\lambda$ but independent of $\delta$.

For any $0<\varepsilon<1 / 2$, choose $\xi_{\varepsilon} \in C^{\infty}([0,1])$ to satisfy

$$
\xi_{\varepsilon}(x)= \begin{cases}0, & \text { if } 0 \leq x \leq \varepsilon, \\ 1, & \text { if } 2 \varepsilon \leq x \leq 1\end{cases}
$$

and

$$
0 \leq \xi_{\varepsilon}(x) \leq 1, \quad 0 \leq \xi_{\varepsilon}^{\prime}(x) \leq \frac{2}{\varepsilon}, \quad\left|\xi_{\varepsilon}^{\prime \prime}(x)\right| \leq \frac{4}{\varepsilon^{2}}, \quad 0 \leq x \leq 1
$$


Assume that $u$ is a solution to problem (1.2)-(1.4) in $(0,+\infty)$. Then, it follows from Definition 2.1 that $u$ satisfies

$$
\begin{aligned}
& \frac{d}{d t} \int_{0}^{1} u(x, t) \xi_{\varepsilon}(x) \psi_{\delta}(x) d x \\
= & \int_{0}^{1} u(x, t)\left(x^{\lambda}\left(\xi_{\varepsilon}(x) \psi_{\delta}(x)\right)^{\prime}\right)^{\prime} d x+\int_{0}^{1} u^{p}(x, t) \xi_{\varepsilon}(x) \psi_{\delta}(x) d x \\
= & \int_{0}^{1} u(x, t) \xi_{\varepsilon}(x)\left(x^{\lambda} \psi_{\delta}^{\prime}(x)\right)^{\prime} d x+\int_{\varepsilon}^{2 \varepsilon} u(x, t) \xi_{\varepsilon}^{\prime}(x)\left(2 x^{\lambda} \psi_{\delta}^{\prime}(x)+\lambda x^{\lambda-1} \psi_{\delta}(x)\right) d x \\
& \quad+\int_{\varepsilon}^{2 \varepsilon} u(x, t) x^{\lambda} \xi_{\varepsilon}^{\prime \prime}(x) \psi_{\delta}(x) d x+\int_{0}^{1} u^{p}(x, t) \xi_{\varepsilon}(x) \psi_{\delta}(x) d x, \quad t>0 .
\end{aligned}
$$

Owing to $\lambda \geq 2,0<\delta<1$ and $u(\cdot, t) \in L^{\infty}((0,1))(t>0)$, letting $\varepsilon \rightarrow 0^{+}$in (3.4) yields

$$
\begin{aligned}
& \frac{d}{d t} \int_{0}^{1} u(x, t) \psi_{\delta}(x) d x=\int_{0}^{1} u(x, t)\left(x^{\lambda} \psi_{\delta}^{\prime}(x)\right)^{\prime} d x+\int_{0}^{1} u^{p}(x, t) \psi_{\delta}(x) d x \\
& \quad \geq \int_{0}^{1} u(x, t)\left(x^{\lambda} \psi_{\delta}^{\prime}(x)\right)^{\prime} d x+\left(\int_{0}^{1} \psi_{\delta}(x) d x\right)^{1-p}\left(\int_{0}^{1} u(x, t) \psi_{\delta}(x) d x\right)^{p}, \quad t>0 .
\end{aligned}
$$

Substitute (3.2) and (3.3) into (3.5) to get

$$
\begin{aligned}
\frac{d}{d t} \int_{0}^{1} u(x, t) \psi_{\delta}(x) d x \geq & -C_{1} \delta \int_{0}^{1} u(x, t) \psi_{\delta}(x) d x \\
& +C_{2}^{1-p}\left(\int_{0}^{1} u(x, t) \psi_{\delta}(x) d x\right)^{p}, \quad t>0 .
\end{aligned}
$$

For nontrivial $u_{0}$, noting $\inf _{0<\delta<1} \int_{0}^{1} u_{0}(x) \psi_{\delta}(x) d x>0$, there exists sufficiently small $0<\delta<1$ such that

$$
2 C_{1} \delta \leq C_{2}^{1-p}\left(\int_{0}^{1} u_{0}(x) \psi_{\delta}(x) d x\right)^{p-1}
$$

Then, (3.6) gives

$$
\frac{d}{d t} \int_{0}^{1} u(x, t) \psi_{\delta}(x) d x \geq \frac{1}{2} C_{2}^{1-p}\left(\int_{0}^{1} u(x, t) \psi_{\delta}(x) d x\right)^{p}, \quad t>0 .
$$

Therefore, there exists $T>0$ such that

$$
\lim _{t \rightarrow T^{-}} \int_{0}^{1} u(x, t) \psi_{\delta}(x) d x=+\infty
$$

which leads to

$$
\lim _{t \rightarrow T^{-}} \sup _{(0,1)} u(\cdot, t)=+\infty
$$

Proof of Theorem 2.3. According to Definition 2.1 for any $0<\tilde{T}<T$, there exists $M>0$ such that

$$
0 \leq u(x, t) \leq M^{1 /(p-1)}, \quad 0<x<1,0<t<\tilde{T} .
$$


First consider case (i). Assume that $u_{0}\left(x_{0}\right) \neq 0$ for some $0<x_{0}<1$. Noting that $u$ is a supersolution to the uniformly parabolic problem

$$
\begin{array}{ll}
\frac{\partial \tilde{u}}{\partial t}=\frac{\partial}{\partial x}\left(x^{\lambda} \frac{\partial \tilde{u}}{\partial x}\right), & \frac{x_{0}}{2}<x<\frac{x_{0}+1}{2}, 0<t<\tilde{T}, \\
\tilde{u}\left(\frac{x_{0}}{2}, t\right)=\tilde{u}\left(\frac{x_{0}+1}{2}, t\right)=0, & 0<t<\tilde{T}, \\
\tilde{u}(x, 0)=u_{0}(x), & \frac{x_{0}}{2}<x<\frac{x_{0}+1}{2},
\end{array}
$$

there exists $\delta>0$ such that

$$
u\left(x_{0}, t\right) \geq \delta, \quad 0<t<\tilde{T} .
$$

Consider the control system

$$
\begin{array}{ll}
\frac{\partial \tilde{v}}{\partial t}=\frac{\partial}{\partial x}\left(x^{\lambda} \frac{\partial \tilde{v}}{\partial x}\right)+h(x, t) \chi_{\left(\left(2 x_{0}+1\right) / 3,\left(x_{0}+2\right) / 3\right),} & 0<x<1,0<t<\tilde{T}, \\
x^{\lambda} \frac{\partial \tilde{v}}{\partial x}(0, t)=0, \quad \tilde{v}(1, t)=0, & 0<t<\tilde{T}, \\
\tilde{v}(x, 0)=v_{0}(x), & 0<x<1,
\end{array}
$$

where $v_{0} \in C^{2}([0,1])$ with $v_{0}(x)=-1$ for $0<x<x_{0}$ and $v_{0}(1)=0$. Since $0<\lambda<2$, there exists $h \in L^{2}((0,1) \times(0, \tilde{T}))$ such that $\tilde{v}(x, T)=0$ for $0<x<1$ (see for example [7, 14]). According to the Moser iteration and the comparison principle, one can show that $\tilde{v} \in L^{\infty}\left(\left(0, x_{0}\right) \times(0, \tilde{T})\right)$. Set

$$
\hat{v}(x, t)=\frac{\delta}{\|\tilde{v}\|_{L^{\infty}\left(\left(0, x_{0}\right) \times(0, \tilde{T})\right)}+1}(\tilde{v}(x, t)+1), \quad 0<x<x_{0}, 0<t<\tilde{T} .
$$

Then, $\hat{v}$ is a subsolution to the problem

$$
\begin{array}{ll}
\frac{\partial v}{\partial t}=\frac{\partial}{\partial x}\left(x^{\lambda} \frac{\partial v}{\partial x}\right), & 0<x<x_{0}, 0<t<\tilde{T}, \\
x^{\lambda} \frac{\partial v}{\partial x}(0, t)=0, \quad v\left(x_{0}, t\right)=\delta, & 0<t<\tilde{T}, \\
v(x, 0)=0, & 0<x<x_{0} .
\end{array}
$$

Due to (3.7) and (3.8), $u$ is a supersolution to problem (3.9)-(3.11). Therefore, the comparison principle leads to

$$
u(x, \tilde{T}) \geq \hat{v}(x, \tilde{T}) \geq \frac{\delta}{\|\tilde{v}\|_{L^{\infty}\left(\left(0, x_{0}\right) \times(0, \tilde{T})\right)}+1}, \quad 0<x<x_{0} .
$$

Let us turn to case (ii). It follows from (3.7) that $u$ is a subsolution to the problem

$$
\begin{array}{ll}
\frac{\partial w}{\partial t}=\frac{\partial}{\partial x}\left(x^{\lambda} \frac{\partial w}{\partial x}\right)+M w, & 0<x<1,0<t<\tilde{T}, \\
x^{\lambda} \frac{\partial w}{\partial x}(0, t)=0, \quad w(1, t)=0, & 0<t<\tilde{T}, \\
w(x, 0)=u_{0}(x), & 0<x<1 .
\end{array}
$$

The comparison principle yields that

$$
u(x, t) \leq w(x, t), \quad 0<x<1,0<t<\tilde{T} .
$$


Consider the regularized problem

$$
\begin{array}{ll}
\frac{\partial w_{n}}{\partial t}=\frac{\partial}{\partial x}\left(\left(x+\frac{1}{n}\right)^{\lambda} \frac{\partial w_{n}}{\partial x}\right)+M w_{n}, & 0<x<1,0<t<\tilde{T}, \\
\frac{\partial w_{n}}{\partial x}(0, t)=0, \quad w_{n}(1, t)=0, & 0<t<\tilde{T}, \\
w_{n}(x, 0)=u_{0, n}(x), & 0<x<1,
\end{array}
$$

where $n$ is a positive integer and $\left\{u_{0, n}\right\}_{n=1}^{\infty} \subset C_{0}^{\infty}([0,1])$ converges to $u_{0}$ in $L^{2}((0,1))$ and satisfies

$$
\begin{gathered}
0 \leq u_{0, n}(x) \leq N x, \quad\left|\left(\left(x+\frac{1}{n}\right)^{\lambda} u_{0, n}^{\prime}(x)\right)^{\prime}\right| \leq N\left(x+\frac{1}{n}\right)^{\lambda-1}, \\
0<x<1, \quad n=1,2, \cdots
\end{gathered}
$$

with $N=2\left\|u_{0}^{\prime \prime}\right\|_{L^{\infty}((0,1))}+\lambda\left\|u_{0}^{\prime}\right\|_{L^{\infty}((0,1))}$. From the classical theory and energy estimate, problem (3.13)-(3.15) uniquely admits a solution $w_{n} \in C^{\infty}([0,1] \times[0, \tilde{T}])$ and there exists a subsequence of $\left\{w_{n}\right\}_{n=1}^{\infty}$ which converges to $w$ in some sense. Note that $\zeta_{n}=\frac{\partial w_{n}}{\partial t}$ is the solution to the problem

$$
\begin{array}{ll}
\frac{\partial \zeta_{n}}{\partial t}=\frac{\partial}{\partial x}\left(\left(x+\frac{1}{n}\right)^{\lambda} \frac{\partial \zeta_{n}}{\partial x}\right)+M \zeta_{n}, & 0<x<1,0<t<\tilde{T}, \\
\frac{\partial \zeta_{n}}{\partial x}(0, t)=0, \quad \zeta_{n}(1, t)=0, & 0<t<\tilde{T}, \\
\zeta_{n}(x, 0)=\left(\left(x+\frac{1}{n}\right)^{\lambda} u_{0, n}^{\prime}(x)\right)^{\prime}, & 0<x<1 .
\end{array}
$$

Set

$\xi_{n}(x, t)=N \mathrm{e}^{M t+\beta_{n} t}\left(\left(x+\frac{1}{n}\right)^{\lambda-1}+(\lambda-1)\left(\frac{1}{n x+1}\right)^{\lambda-1}\right), \quad 0<x<1,0<t<\tilde{T}$, where $\beta_{n}=2(\lambda-1)^{2}(1+1 / n)^{\lambda-2}$. Then,

$$
\frac{\partial \xi_{n}}{\partial x}(0, t)=N(\lambda-1) \mathrm{e}^{M t+\beta_{n} t}\left(\frac{1}{n^{\lambda-2}}-(\lambda-1) n\right) \leq 0, \quad 0<t<\tilde{T},
$$

and

$$
\begin{aligned}
& \frac{\partial \xi_{n}}{\partial t}-\frac{\partial}{\partial x}\left(\left(x+\frac{1}{n}\right)^{\lambda} \frac{\partial \xi_{n}}{\partial x}\right)-M \xi_{n} \\
&= N \mathrm{e}^{M t+\beta_{n} t}\left(\left(x+\frac{1}{n}\right)^{\lambda-1}\left(\beta_{n}-2(\lambda-1)^{2}\left(x+\frac{1}{n}\right)^{\lambda-2}\right)\right. \\
&+N \beta_{n}(\lambda-1) \mathrm{e}^{M t+\beta_{n} t}\left(\frac{1}{n x+1}\right)^{\lambda-1} \\
& \geq 0, \quad 0<x<1,0<t<\tilde{T},
\end{aligned}
$$

owing to $\lambda \geq 2$. Therefore, it can be verified that $\pm \xi_{n}$ are super and subsolutions to problem (3.16)-(3.18), respectively. The comparison principle yields

$$
\begin{gathered}
\left|\frac{\partial w_{n}}{\partial t}(x, t)\right| \leq N \mathrm{e}^{M t+\beta_{n} t}\left(\left(x+\frac{1}{n}\right)^{\lambda-1}+(\lambda-1)\left(\frac{1}{n x+1}\right)^{\lambda-1}\right), \\
0<x<1,0<t<\tilde{T},
\end{gathered}
$$

which, together with (3.15), leads to the fact that for any $(x, t) \in(0,1) \times(0, \tilde{T})$, $w_{n}(x, t) \leq u_{0, n}(x)+\frac{N}{M+\beta_{n}}\left(\mathrm{e}^{M t+\beta_{n} t}-1\right)\left(\left(x+\frac{1}{n}\right)^{\lambda-1}+(\lambda-1)\left(\frac{1}{n x+1}\right)^{\lambda-1}\right)$. 
Hence

$$
\begin{aligned}
w(x, t) & \leq N x+\frac{N}{M+2(\lambda-1)^{2}}\left(\mathrm{e}^{M t+2(\lambda-1)^{2} t}-1\right) x^{\lambda-1} \\
& \leq 2 N \mathrm{e}^{M t+2(\lambda-1)^{2} t} x, \quad 0<x<1,0<t<\tilde{T}
\end{aligned}
$$

owing to $\lambda \geq 2$. It follows from (3.7), (3.12) and (3.19) that

$$
0 \leq u(x, t) \leq 2 N \mathrm{e}^{M t+2(\lambda-1)^{2} t} x, \quad 0<x<1,0<t<\tilde{T} .
$$

\section{Problems in an unbounded interval}

In this section, we study problem (1.5)-(1.7). Note that Theorem 2.5 is a corollary of Theorem 2.2, while Theorem 2.6 can be proved by a similar proof in Theorem 2.3. Thus we only need it to prove Theorem 2.4.

Proof of Theorem 2.4 (i) and (iii). For $R>0$, set

$$
\psi_{R}(x)= \begin{cases}1, & \text { if } 0<x \leq R, \\ \frac{1}{2}\left(1+\cos \frac{(x-R) \pi}{R}\right), & \text { if } R<x<2 R, \\ 0, & \text { if } x \geq 2 R .\end{cases}
$$

It is clear that $\psi_{R} \in C^{1}([0,+\infty))$ is piecewise smooth with

$$
\begin{gathered}
\left(x^{\lambda} \psi_{R}^{\prime}(x)\right)^{\prime}=-\frac{\lambda \pi}{2 R} x^{\lambda-1} \sin \frac{(x-R) \pi}{R}-\frac{\pi^{2}}{2 R^{2}} x^{\lambda} \cos \frac{(x-R) \pi}{R} \geq-2^{\lambda} \pi^{2} R^{\lambda-2} \psi_{R}(x), \\
R<x<2 R .
\end{gathered}
$$

Assume that $u$ is a solution to problem (1.5) (1.7) in $(0,+\infty)$. Then, it follows from Definition 2.2 that $u$ satisfies

$$
\frac{d}{d t} \int_{0}^{+\infty} u(x, t) \psi_{R}(x) d x=\int_{0}^{+\infty} u(x, t)\left(x^{\lambda} \psi_{R}^{\prime}(x)\right)^{\prime} d x+\int_{0}^{+\infty} u^{p}(x, t) \psi_{R}(x) d x, \quad t>0 .
$$

Therefore,

$$
\begin{aligned}
& \frac{d}{d t} \int_{0}^{+\infty} u(x, t) \psi_{R}(x) d x \\
& \geq-2^{\lambda} \pi^{2} R^{\lambda-2} \int_{0}^{+\infty} u(x, t) \psi_{R}(x) d x+\left(\int_{0}^{+\infty} \psi_{R}(x) d x\right)^{1-p}\left(\int_{0}^{+\infty} u(x, t) \psi_{R}(x) d x\right)^{p} \\
& \geq-2^{\lambda} \pi^{2} R^{\lambda-2} \int_{0}^{+\infty} u(x, t) \psi_{R}(x) d x+2^{1-p} R^{1-p}\left(\int_{0}^{+\infty} u(x, t) \psi_{R}(x) d x\right)^{p}, \quad t>0 .
\end{aligned}
$$

In case (i), since $1<p<3-\lambda$ and $u_{0}$ is nontrivial, there exists $R>0$ sufficiently large such that

$$
2^{\lambda+1} \pi^{2} R^{\lambda-2} \leq 2^{1-p} R^{1-p}\left(\int_{0}^{+\infty} u_{0}(x) \psi_{R}(x) d x\right)^{p-1} .
$$

Then, (4.3) leads to

$$
\frac{d}{d t} \int_{0}^{+\infty} u(x, t) \psi_{R}(x) d x \geq 2^{-p} R^{1-p}\left(\int_{0}^{+\infty} u(x, t) \psi_{R}(x) d x\right)^{p}, \quad t>0 .
$$


Therefore, there exists $T>0$ such that

$$
\lim _{t \rightarrow T^{-}} \int_{0}^{+\infty} u(x, t) \psi_{R}(x) d x=+\infty,
$$

which leads to

$$
\lim _{t \rightarrow T^{-}} \sup _{(0,+\infty)} u(\cdot, t)=+\infty .
$$

Turn to case (iii) where $p>3-\lambda$. From Theorem 2.1 and the comparison principle (Proposition 2.1 (ii)), problem (1.5)-(1.7) admits blowing-up solutions. Let us show there also exist nontrivial global solutions to problem (1.5)-(1.7). Set

$$
\bar{u}(x, t)=(t+1)^{-1 /(p-1)} w\left((t+1)^{-1 /(2-\lambda)} x\right), \quad x>0, t>0 .
$$

A direct calculation shows that $\bar{u}$ is a supersolution to (1.5) if $w \in C^{2}((0,+\infty))$ satisfies

$$
\left(r^{\lambda} w^{\prime}(r)\right)^{\prime}+\frac{1}{2-\lambda} r w^{\prime}(r)+\frac{1}{p-1} w(r)+w^{p}(r) \leq 0, \quad r>0 .
$$

Take

where $\varepsilon>0$ and

$$
w(r)=\varepsilon \mathrm{e}^{-A r^{2-\lambda}}, \quad r>0,
$$

Then,

$$
\frac{1}{(2-\lambda)(p-1)}<A<\frac{1}{(2-\lambda)^{2}}
$$

$$
\begin{gathered}
\left(r^{\lambda} w^{\prime}(r)\right)^{\prime}+\frac{1}{2-\lambda} r w^{\prime}(r)+\frac{1}{p-1} w(r)+w^{p}(r) \\
=\varepsilon \mathrm{e}^{-A r^{2-\lambda}}\left(A(2-\lambda)\left(A(2-\lambda)-\frac{1}{2-\lambda}\right) r^{2-\lambda}\right. \\
\left.\quad+\left(\frac{1}{p-1}-A(2-\lambda)\right)+\varepsilon^{p-1} \mathrm{e}^{-A(p-1) r^{2-\lambda}}\right) \\
\leq \varepsilon \mathrm{e}^{-A r^{2-\lambda}}\left(\begin{array}{r}
A(2-\lambda)\left(A(2-\lambda)-\frac{1}{2-\lambda}\right) r^{2-\lambda} \\
\quad
\end{array}\right. \\
\left.\quad\left(\frac{1}{p-1}-A(2-\lambda)\right)+\varepsilon^{p-1}\right), \quad r>0
\end{gathered}
$$

From the range of $A$,

$$
A(2-\lambda)<\frac{1}{2-\lambda}, \quad \frac{1}{p-1}<A(2-\lambda) .
$$

Hence $\bar{u}$ is a supersolution to (1.5) for each $0<\varepsilon \leq \varepsilon_{0}$, where

$$
\varepsilon_{0}=\left(A(2-\lambda)-\frac{1}{p-1}\right)^{1 /(p-1)} .
$$

Noting $\lim _{r \rightarrow 0^{+}} r^{\lambda} w^{\prime}(r)=0$, one gets that $\bar{u}$ is a supersolution to problem (1.5)(1.7) if

$$
u_{0}(x) \leq \bar{u}(x, 0), \quad x>0 \quad\left(0<\varepsilon \leq \varepsilon_{0}\right) .
$$

The comparison principle (Proposition 2.2 (ii)) yields that the solution to problem (1.5) - (1.7) exists globally in time if $u_{0}$ satisfies (4.4). 
In order to prove Theorem 2.4 (ii), we need the following lemma.

Lemma 4.1. Let $0<\lambda<2, p=3-\lambda$ and $u$ be the solution to problem (1.5) (1.7) in $(0,+\infty)$. Then for any $R>0$,

$$
\begin{gathered}
\int_{0}^{+\infty} u(x, t) \psi_{R}(x) d x \leq 2^{3 /(2-\lambda)} \pi^{2 /(2-\lambda)}, \quad t>0, \\
\frac{d}{d t} \int_{0}^{+\infty} u(x, t) \psi_{R}(x) d x \geq-2^{\lambda+2 /(2-\lambda)} \pi^{2(3-\lambda) /(2-\lambda)} R^{\lambda-2}, \quad t>0,
\end{gathered}
$$

and

$$
\begin{aligned}
& \frac{d}{d t} \int_{0}^{+\infty} u(x, t) \psi_{R}(x) d x \\
& \quad \geq R^{\lambda-2}\left(\int_{0}^{+\infty} u(x, t) \psi_{R}(x) d x\right)^{1 / 2}\left(-2^{\lambda} \pi^{2}\left(\int_{R}^{2 R} u(x, t) \psi_{R}(x) d x\right)^{1 / 2}\right. \\
& \left.\quad+2^{\lambda-2}\left(\int_{0}^{+\infty} u(x, t) \psi_{R}(x) d x\right)^{5 / 2-\lambda}\right), \quad t>0,
\end{aligned}
$$

where $\psi_{R}$ is the function defined by (4.1).

Proof. First, one gets from (4.3) that

$$
\begin{aligned}
& \frac{d}{d t} \int_{0}^{+\infty} u(x, t) \psi_{R}(x) d x \\
& \geq-2^{\lambda} \pi^{2} R^{\lambda-2} \int_{0}^{+\infty} u(x, t) \psi_{R}(x) d x+2^{\lambda-2} R^{\lambda-2}\left(\int_{0}^{+\infty} u(x, t) \psi_{R}(x) d x\right)^{3-\lambda}, \quad t>0 .
\end{aligned}
$$

Let us prove (4.5) by a contradiction. Otherwise, there exists $t_{0}>0$ such that

$$
2^{\lambda+1} \pi^{2} \geq 2^{\lambda-2}\left(\int_{0}^{+\infty} u\left(x, t_{0}\right) \psi_{R}(x) d x\right)^{2-\lambda}
$$

Then, it follows from (4.8) that

$$
\frac{d}{d t} \int_{0}^{+\infty} u(x, t) \psi_{R}(x) d x \geq 2^{\lambda-3} R^{\lambda-2}\left(\int_{0}^{+\infty} u(x, t) \psi_{R}(x) d x\right)^{3-\lambda}, \quad t>t_{0},
$$

which leads to the fact that $u$ must blow up in a finite time. Thus, (4.5) holds. Second, from (4.8) and the Young inequality, one can get that

$$
\begin{aligned}
& \frac{d}{d t} \int_{0}^{+\infty} u(x, t) \psi_{R}(x) d x \\
& \geq 2^{\lambda-2} R^{\lambda-2}\left(-4 \pi^{2} \int_{0}^{+\infty} u(x, t) \psi_{R}(x) d x+\left(\int_{0}^{+\infty} u(x, t) \psi_{R}(x) d x\right)^{3-\lambda}\right) \\
& \geq 2^{\lambda-2} R^{\lambda-2}\left(-\frac{1}{3-\lambda}\left(\int_{0}^{+\infty} u(x, t) \psi_{R}(x) d x\right)^{3-\lambda}-\frac{2-\lambda}{3-\lambda}\left(4 \pi^{2}\right)^{(3-\lambda) /(2-\lambda)}\right. \\
& \left.+\left(\int_{0}^{+\infty} u(x, t) \psi_{R}(x) d x\right)^{3-\lambda}\right) \\
& \geq-2^{\lambda+2 /(2-\lambda)} \pi^{2(3-\lambda) /(2-\lambda)} R^{\lambda-2}, \quad t>0 .
\end{aligned}
$$


Finally, it follows from (4.2) that

$$
\begin{aligned}
& \frac{d}{d t} \int_{0}^{+\infty} u(x, t) \psi_{R}(x) d x \\
& =\int_{R}^{2 R} u(x, t)\left(x^{\lambda} \psi_{R}^{\prime}(x)\right)^{\prime} d x+\int_{0}^{+\infty} u^{3-\lambda}(x, t) \psi_{R}(x) d x \\
& \geq-2^{\lambda} \pi^{2} R^{\lambda-2} \int_{R}^{2 R} u(x, t) \psi_{R}(x) d x \\
& \quad+\left(\int_{0}^{+\infty} \psi_{R}(x) d x\right)^{\lambda-2}\left(\int_{0}^{+\infty} u(x, t) \psi_{R}(x) d x\right)^{3-\lambda} \\
& \geq-2^{\lambda} \pi^{2} R^{\lambda-2} \int_{R}^{2 R} u(x, t) \psi_{R}(x) d x+2^{\lambda-2} R^{\lambda-2}\left(\int_{0}^{+\infty} u(x, t) \psi_{R}(x) d x\right)^{3-\lambda} \\
& \geq R^{\lambda-2}\left(\int_{0}^{+\infty} u(x, t) \psi_{R}(x) d x\right)^{1 / 2}\left(-2^{\lambda} \pi^{2}\left(\int_{R}^{2 R} u(x, t) \psi_{R}(x) d x\right)^{1 / 2}\right. \\
& \left.\quad+2^{\lambda-2}\left(\int_{0}^{+\infty} u(x, t) \psi_{R}(x) d x\right)^{5 / 2-\lambda}\right), \quad t>0 .
\end{aligned}
$$

Proof of Theorem 2.4 (ii). Assume that $u$ is a solution to problem (1.5)-(1.7) in $(0,+\infty)$. For any $R>0$, set

$$
w_{R}(t)=\int_{0}^{+\infty} u(x, t) \psi_{R}(x) d x, \quad t \geq 0,
$$

where $\psi_{R}$ is the function defined by (4.1). Denote

$$
\Lambda=\sup _{R>0, t>0} w_{R}(t)=\sup _{t>0} \int_{0}^{+\infty} u(x, t) d x .
$$

Then $0<\Lambda<+\infty$ due to (4.5) and the nontriviality of $u_{0}$. Fix $\varepsilon_{0} \in(0, \Lambda)$ and $M_{0}>0$ such that

$$
2^{\lambda} \pi^{2}\left(\varepsilon_{0}+M_{0}\right)^{1 / 2} \leq 2^{\lambda-3}\left(\Lambda-\varepsilon_{0}\right)^{5 / 2-\lambda} .
$$

Owing to (4.9), there exists $t_{1} \geq 0$ and $R_{0}>0$ such that

$$
w_{R_{0}}\left(t_{1}\right) \geq \Lambda-\varepsilon_{0} \text {. }
$$

For any $t \geq t_{1}$, it follows from (4.6) with $R=R_{0}$ and (4.10) that

$$
\begin{aligned}
\int_{0}^{+\infty} & u(x, t) \psi_{R_{0}}(x) d x \\
\quad \geq & \int_{0}^{+\infty} u\left(x, t_{1}\right) \psi_{R_{0}}(x) d x-2^{\lambda+2 /(2-\lambda)} \pi^{2(3-\lambda) /(2-\lambda)} R_{0}^{\lambda-2}\left(t-t_{1}\right) \\
\quad \geq & \Lambda-\varepsilon_{0}-2^{\lambda+2 /(2-\lambda)} \pi^{2(3-\lambda) /(2-\lambda)} R_{0}^{\lambda-2}\left(t-t_{1}\right),
\end{aligned}
$$

which, together with (4.9), leads to

$$
\begin{aligned}
\int_{2 R_{0}}^{4 R_{0}} u(x, t) \psi_{2 R_{0}}(x) d x & \leq \int_{0}^{+\infty} u(x, t) d x-\int_{0}^{+\infty} u(x, t) \psi_{R_{0}}(x) d x \\
& \leq \varepsilon_{0}+2^{\lambda+2 /(2-\lambda)} \pi^{2(3-\lambda) /(2-\lambda)} R_{0}^{\lambda-2}\left(t-t_{1}\right) .
\end{aligned}
$$


Choosing $R=2 R_{0}$ in (4.7) yields

$$
\begin{aligned}
\frac{d}{d t} w_{2 R_{0}}(t) \geq\left(2 R_{0}\right)^{\lambda-2} w_{2 R_{0}}^{1 / 2}(t)\left(-2^{\lambda} \pi^{2}\left(\int_{2 R_{0}}^{4 R_{0}} u(x, t) \psi_{2 R_{0}}(x) d x\right)^{1 / 2}\right. & \\
& \left.+2^{\lambda-2} w_{2 R_{0}}^{5 / 2-\lambda}(t)\right), \quad t>t_{1},
\end{aligned}
$$

which, together with (4.9)-4.11), implies

$$
\frac{d}{d t} w_{2 R_{0}}(t) \geq 2^{2 \lambda-5} R_{0}^{\lambda-2}\left(\Lambda-\varepsilon_{0}\right)^{3-\lambda}, \quad t_{1}<t<t_{2},
$$

where

$$
t_{2}=t_{1}+2^{-\lambda-2 /(2-\lambda)} \pi^{-2(3-\lambda) /(2-\lambda)} M_{0} R_{0}^{2-\lambda} .
$$

Then, it follows from (4.12) and (4.10) that

$$
w_{2 R_{0}}\left(t_{2}\right) \geq w_{2 R_{0}}\left(t_{1}\right)+2^{2 \lambda-5} R_{0}^{\lambda-2}\left(\Lambda-\varepsilon_{0}\right)^{3-\lambda}\left(t_{2}-t_{1}\right) \geq \Lambda-\varepsilon_{0}+\gamma_{0},
$$

where

$$
\gamma_{0}=2^{\lambda-5-2 /(2-\lambda)} \pi^{-2(3-\lambda) /(2-\lambda)} M_{0}\left(\Lambda-\varepsilon_{0}\right)^{3-\lambda} .
$$

Repeating the procedure in turn, one can get that for any positive integer $i$,

$$
w_{2^{i} R_{0}}\left(t_{i+1}\right) \geq w_{2^{i} R_{0}}\left(t_{i}\right)+\gamma_{0} \geq w_{2^{i-1} R_{0}}\left(t_{i}\right)+\gamma_{0} \geq \Lambda-\varepsilon_{0}+i \gamma_{0},
$$

where

$$
t_{i+1}=t_{i}+2^{-\lambda-2 /(2-\lambda)} \pi^{-2(3-\lambda) /(2-\lambda)} M_{0}\left(2^{i-1} R_{0}\right)^{2-\lambda} .
$$

Hence

$$
\sup _{t>0} \int_{0}^{+\infty} u(x, t) d x=+\infty
$$

which contradicts (4.9) and completes the proof of Theorem 2.4 (ii).

\section{Problems With inNer Degeneracy}

In this section, we consider the problems

$$
\begin{array}{ll}
\frac{\partial u}{\partial t}=\frac{\partial}{\partial x}\left(|x|^{\lambda} \frac{\partial u}{\partial x}\right)+u^{p}, & -1<x<1, t>0, \\
u( \pm 1, t)=0, & t>0, \\
u(x, 0)=u_{0}(x), & -1<x<1
\end{array}
$$

and

$$
\begin{array}{ll}
\frac{\partial u}{\partial t}=\frac{\partial}{\partial x}\left(|x|^{\lambda} \frac{\partial u}{\partial x}\right)+u^{p}, & x \in \mathbb{R}, t>0, \\
u(x, 0)=u_{0}(x), & x \in \mathbb{R},
\end{array}
$$

where $\lambda>0, p>1$ and $u_{0}$ is nonnegative. For these problems with inner degeneracy, the definitions of solutions, the well-posedness and the comparison principles are similar to problem (1.2)-(1.4) and problem (1.5)-(1.7). Furthermore, by the same proofs as in $\S 3$ and $\S 4$, we can prove the following theorems.

Theorem 5.1. Assume that $0<\lambda<2$. Then there exist both nontrivial global and blowing-up solutions to problem (5.1) (5.3). 
Theorem 5.2. Assume that $\lambda \geq 2$. Then the solution to problem (5.1)-(5.3) must blow up in a finite time for any nontrivial $0 \leq u_{0} \in L^{\infty}((-1,1))$ with $|x|^{\lambda / 2} u_{0}^{\prime} \in$ $L^{2}((-1,1))$.

Theorem 5.3. Assume that $u$ is the solution to problem (5.1)-(5.3) in $(0, T)$ $(0<T \leq+\infty)$ for nontrivial $0 \leq u_{0} \in C^{2}([-1,1])$ with $u_{0}(0)=0$.

(i) If $0<\lambda<2$, then

$$
\lim _{x \rightarrow 0} u(x, t)>0, \quad 0<t<T .
$$

(ii) If $\lambda \geq 2$, then for any $0<t<T$, there exists $M(t)>0$ such that

$$
0 \leq u(x, t) \leq M(t)|x|, \quad-1<x<1 .
$$

Theorem 5.4. Assume that $0<\lambda<2$.

(i) If $1<p \leq 3-\lambda$, then the solution to problem (5.4), (5.5) must blow up in a finite time for any nontrivial $0 \leq u_{0} \in L^{\infty}(\mathbb{R})$ with $|x|^{\lambda / 2} u_{0}^{\prime} \in L_{\mathrm{loc}}^{2}(\mathbb{R})$.

(ii) If $p>3-\lambda$, then there exist both nontrivial global and blowing-up solutions to problem (5.4), (5.5).

Theorem 5.5. Assume that $\lambda \geq 2$. Then the solution to problem (5.4), (5.5) must blow up in a finite time for any nontrivial $0 \leq u_{0} \in L^{\infty}(\mathbb{R})$ with $|x|^{\lambda / 2} u_{0}^{\prime} \in L_{\text {loc }}^{2}(\mathbb{R})$.

Theorem 5.6. Assume that $\lambda \geq 2$ and $u$ is the solution to problem (5.4), (15.5) in $(0, T)(0<T \leq+\infty)$ for nontrivial $0 \leq u_{0} \in C^{2}(\mathbb{R}) \cap L^{\infty}(\mathbb{R})$ with $u_{0}(0)=0$.

(i) If $0<\lambda<2$, then

$$
\frac{\lim _{x \rightarrow 0} u(x, t)>0, \quad 0<t<T .}{0}
$$

(ii) If $\lambda \geq 2$, then for any $0<t<T$, there exists $M(t)>0$ such that

$$
0 \leq u(x, t) \leq M(t)|x|, \quad x \in \mathbb{R} .
$$

\section{REFERENCES}

[1] Alabau-Boussouira, F., Cannarsa, P. and Fragnelli, G., Carleman estimates for degenerate parabolic operators with applications to null controllability, J. Evol. Equ., 6(2)(2006), 161-204. MR2227693 (2007e:93010)

[2] Black, F. and Scholes, M., The pricing of options and corporate liabilities, Journal of Political Economy, 81(1973), 637-659.

[3] Cannarsa, P., Fragnelli, G. and Vancostenoble, J., Linear degenerate parabolic equations in bounded domains: Controllability and observability, systems, control, modeling and optimization, 163-173, IFIP Int. Fed. Inf. Process., 202, Springer, New York, 2006. MR2241704(2007e:93013)

[4] Cannarsa, P., Fragnelli, G. and Vancostenoble, J., Regional controllability of semilinear degenerate parabolic equations in bounded domains, J. Math. Anal. Appl., 320(2)(2006), 804818. MR2225996(2007a:93006)

[5] Cannarsa, P., Martinez, P. and Vancostenoble, J., Persistent regional null controllability for a class of degenerate parabolic equations, Commun. Pure Appl. Anal., 3(4)(2004), 607635. MR2106292 (2005i:93011)

[6] Cannarsa, P., Martinez, P. and Vancostenoble J., Null controllability of degenerate heat equations, Adv. Differential Equations, 10(2)(2005), 153-190. MR2106129 (2005j:93010)

[7] Cannarsa, P., Martinez, P. and Vancostenoble, J., Carleman estimates for a class of degenerate parabolic operators, SIAM J. Control Optim., 47(1)(2008), 1-19. MR2373460 (2009i:35033)

[8] Deng, K. and Levine, H. A., The role of critical exponents in blow-up theorems: The sequel, J. Math. Anal. Appl., 243(1)(2000), 85-126. MR1742850(2001b:35031) 
[9] Fusita, H., On the blowing up of solutions of the Cauchy problem for $u_{t}=\Delta u+u^{1+\alpha}$, J. Fac. Sci. Univ. Tokyo Sect. I, 13(1966), 109-124. MR0214914 (35:5761)

[10] Levine, H. A., The role of critical exponents in blow-up theorems, SIAM Rev., 32(2)(1990), 262-288. MR 1056055 (91j:35135)

[11] Martinez, P. and Vancostenoble, J., Carleman estimates for one-dimensional degenerate heat equations, J. Evol. Equ., 6(2)(2006), 325-362. MR2227700 (2007a:93016)

[12] North, G. R., Howard, L., Pollard, D. and Wielicki, B., Variational formulation of Budyko-Sellers climate models, J. Atmos. Sci., 36(1979), 255-259.

[13] Wang, C., Approximate controllability of a class of semilinear systems with boundary degeneracy, Journal of Evolution Equations, 10(1)(2010), 163-193. MR2602931 (2011g:93018)

[14] WeI, Y. and Du, R., Null controllability of a class of parabolic systems with boundary degeneracy (Chinese), J. Jilin Univ. Sci., 49(5)(2011), 849-852. MR2885035 (2012i:93028)

[15] Wu, Z., Zhao, J., Yin, J. and Li, H., Nonlinear diffusion equations, World Scientific Publishing Co., Inc., River Edge, NJ, 2001. MR1881297 (2003e:35002)

[16] Yin, J. and Wang, C., Properties of the boundary flux of a singular diffusion process, Chinese Ann. Math. Ser. B, 25(2)(2004), 175-182. MR2055268 (2005c:35166)

[17] Yin, J. and WANG, C., Evolutionary weighted p-Laplacian with boundary degeneracy, J. Differential Equations, 237(2)(2007), 421-445. MR2330953 (2009e:35159)

School of Mathematics, Jilin University, Changchun 130012, People's Republic of China

E-mail address: wangcp@jlu.edu.cn 